\title{
Integrative genomic analyses of a novel cytokine, interleukin-34 and its potential role in cancer prediction
}

\author{
BO WANG ${ }^{1}$, WENMING XU $^{2}$, MIAOLIAN TAN ${ }^{1}$, YAN XIAO $^{1}$, HAIWEI YANG $^{3}$ and TIAN-SONG XIA $^{4}$ \\ Departments of ${ }^{1}$ Medical Oncology and ${ }^{2}$ Endocrinology, The Eastern Hospital of The First Affiliated Hospital, \\ Sun Yat-Sen University, Guangzhou, Guangdong 510700; Departments of ${ }^{3}$ Urology and ${ }^{4}$ Breast Surgery, \\ The First Affiliated Hospital of Nanjing Medical University, Nanjing, Jiangsu 210029, P.R. China
}

Received July 8, 2014; Accepted November 3, 2014

DOI: $10.3892 /$ ijmm.2014.2001

\begin{abstract}
Interleukin-34 (IL-34) is a novel cytokine, which is composed of 222 amino acids and forms homodimers. It binds to the macrophage colony-stimulating factor (M-CSF) receptor and plays an important role in innate immunity and inflammatory processes. In the present study, we identified the completed IL-34 gene in 25 various mammalian genomes and found that IL-34 existed in all types of vertebrates, including fish, amphibians, birds and mammals. These species have a similar 7 exon/6 intron gene organization. The phylogenetic tree indicated that the IL-34 gene from the primate lineage, rodent lineage and teleost lineage form a species-specific cluster. It was found mammalian that IL-34 was under positive selection pressure with the identified positively selected site, 196Val. Fifty-five functionally relevant single nucleotide polymorphisms (SNPs), including 32 SNPs causing missense mutations, 3 exonic splicing enhancer SNPs and 20 SNPs causing nonsense mutations were identified from 2,141 available SNPs in the human IL-34 gene. IL-34 was expressed in various types of cancer, including blood, brain, breast, colorectal, eye, head and neck, lung, ovarian and skin cancer. A total of 5 out of 40 tests ( 1 blood cancer, 1 brain cancer, 1 colorectal cancer and 2 lung cancer) revealed an association between IL-34 gene expression and cancer prognosis. It was found that the association between the expression of IL-34 and cancer prognosis varied in different types of cancer, even in the same types of cancer from different databases. This suggests
\end{abstract}

Correspondence to: Dr Bo Wang, Department of Medical Oncology, The Eastern Hospital of The First Affiliated Hospital, Sun Yat-Sen University, No. 183, Huangpu East Road, Guangzhou, Guangdong 510700, P.R. China

E-mail: wangb68377@sina.com

Dr Tian-Song Xia, Department of Breast Surgery, The First Affiliated Hospital of Nanjing Medical University, No. 300, Guangzhou Road, Nanjing, Jiangsu 210029, P.R. China

E-mail: xiatsswms@163.com

Key words: interleukin-34, comparative genomics, cancer, prognosis, meta-analysis that the function of IL-34 in these tumors may be multidimensional. The upstream transcription factor 1 (USF1), regulatory factor X-1 (RFX1), the Sp1 transcription factor 1 , POU class 3 homeobox 2 (POU3F2) and forkhead box L1 (FOXL1) regulatory transcription factor binding sites were identified in the IL-34 gene upstream (promoter) region, which may be involved in the effects of IL-34 in tumors.

\section{Introduction}

Cytokines are glycosylated proteins that allow communication among various cell types involved in immune response. Interleukins (ILs) are cytokines mainly produced by T-cells, as well by monocytes, macrophages and endothelial cells $(1,2)$. The different ILs share special biochemical or functional characteristics and are numbered in order of their identification. The emergence of new technologies is translating into a steady increase in the number of known molecules (3). In 2008, Lin et al (4) produced 3,400 recombinant secreted proteins that encode secreted proteins and extracellular domains of transmembrane proteins in $293 \mathrm{~T}$ cells and examined their activities based on human monocyte screening assays. Subsequently, the authors (4) discovered a novel cytokine, IL-34. The human IL-34 protein is composed of 222 amino acids, has a molecular mass of $39 \mathrm{kDa}$ and forms homodimers. It binds to the macrophage colony-stimulating factor (M-CSF) receptor, c-FMS (also known as CSF-1 receptor), expressed on the cell surface of human monocytes and has a stronger, although short-lived effect compared to M-CSF. IL-34 has been shown to be involved in the process of osteoclastogenesis and rheumatoid arthritis (RA) (5-8). IL-34 has been shown to promote the proliferation, survival and differentiation of monocytes and macrophages, the release of pro-inflammatory chemokines, and thereby plays an important role in innate immunity and inflammatory processes. It also plays an important role in the regulation of osteoclast proliferation and differentiation, and in the regulation of bone resorption (5-8).

IL-34 and M-CSF both signal via the same receptor, the M-CSF receptor. Although IL-34 and M-CSF show no appreciable similarity in their primary structure, they are evolutionally distant ligands, but are structurally related (9). There is evidence indicating that the M-CSF-IL-34-c-FMS axis is involved in the initiation, growth and metastasis of 
tumors (10,11). M-CSF levels may constitute a useful biomarker for a number of types of cancer, as it is expressed at high levels in a number of types of cancer, including breast cancer, ovarian cancer and colorectal carcinoma and its expression correlates with a poor prognosis (12). The direct inhibition of M-CSF or the inhibition of c-FMS kinase activity can lead to significant changes in the growth of grafted tumors (13.14). Tumorassociated macrophages are the most abundant component of the leukocyte infiltrate of solid tumors. In M-CSF-deficient mice $\left(\mathrm{M}-\mathrm{CSF}^{\mathrm{op} / \mathrm{op}}\right.$ or $\mathrm{M}-\mathrm{CSF}^{-/-}$), the growth of the primary tumor and the metastatic spread of tumor cells has been shown to be significantly reduced due to the inability of angiogenesis to feed the tumors (12-15).

However, studies on the role of IL-34 in tumorigenesis. In the present study, we identified the IL-34 gene in various mammalian genomes by comparative genomic analyses. The conserved transcription factor-binding sites within the promoter region of the human IL-34 gene were then searched. Analyses of the expression data, functional relevant single nucleotide polymorphisms (SNPs) and comparative proteomic analysis were also conducted. Furthermore, a meta-analysis of the prognostic value of the IL-34 gene in various types of cancer was performed.

\section{Materials and methods}

Identification of the novel IL-34 gene in vertebrate genomes and integrative genomic analyses. All the IL-34 gene and amino acid sequences were obtained from the Ensembl database (http://www.ensembl.org/index.html), based on orthologous and paralogous relationships. The gained IL-34 sequences were applied as queries to search the IL-34 gene using BLAST at the National Center for Biotechnology Information (NCBI), in order to confirm whether their best hit was an IL-34 gene (16-18). The number and length of IL-34 exons and introns in all competent sequences were investigated for exon-intron conservation analyses. The number, length and structures of the exons and introns in IL-34 in all species were also collected from the Ensembl database (http://www. ensembl.org/index.html). Conserved transcription factorbinding sites within the promoter region of the human IL-34 gene were obtained from SABiosciences' proprietary database which combines Text Mining Application and data from the UCSC Genome Browser (19-21).

Comparative proteomic analysis of IL-34 protein. The protein coding sequences of IL-34 were aligned using ClustalW software implemented in MEGA 5.05. We constructed a maximum likelihood (ML) tree of IL-34 amino acid sequences using MEGA 5.05 with the optimal model (Kimura 2-parameter model). For the relative support of the internal node, bootstrap analysis was performed with 1,000 replications for ML reconstructions (22). The program CodeML implemented in the PAML 4.7 software package was used to investigate whether the IL-34 protein is under positive selection (23). The site-specific model was exerted using likelihood ratio tests (LRTs) to compare the M7 (null model) with the M8 model. M7 is a null model that does not allow for any codons with $\omega>1$, whereas the M8 model allows for positively selective sites $(\omega>1)$. When the M8 model was fitted to the data more efficiently (P-value $<0.05$ ) than the null model (M7), the presence of sites with $\omega>1$ was suggested. On the contrary, the results of P-value $>0.05$ proved the absence of sites with $\omega>1$. Twice the log likelihood difference between the two compared models $(2 \Delta \mathrm{l})$ was compared against $\chi^{2}$ with critical values of 5.99 and 9.21 at 0.05 and 0.01 significance levels, respectively, as previously described (24).

Functionally relevant SNP evaluation of the human IL-34 gene and identification of somatic mutations in human cancer. Functionally relevant SNPs of the human IL-34 gene were identified as previously described (16-21). The SNPs were extracted from the Ensembl (http://www.ensembl.org) and the NCBI SNPdb (http://www.ncbi.nlm.nih.gov) databases. The SNPs that disrupted exonic splicing enhancer/exonic splicing silencer (ESE/ESS) motifs and cause missence mutations were also identified. The identification of somatic mutations of the human IL-34 gene in human cancer was conducted in the Catalogue of Somatic Mutations in Cancer (COSMIC), a database for mining complete cancer genomes in the catalogue of somatic mutations in cancer (25).

In silico expression analyses of the human IL-34 gene. Expressed sequence tags (ESTs) derived from the human IL-34 gene were searched for using the BLAST programs as previously described (26-29). The human IL-34 gene (NM_152456) was used as query sequences for the BLAST programs. The expression profiles for normal human tissues were obtained from GeneAnnot (30) and ArrayExpress (31) databases. Northern analysis of the NCBI uniGene dataset was also performed (19-21).

Meta-analysis of the prognostic value of the IL-34 gene in cancer. A database termed 'PrognoScan' has been previously developed (32). This database includes a large collection of publicly available cancer microarray datasets with clinical annotation, as well as a tool for assessing the biological association between gene expression and prognosis. PrognoScan employs the minimum P-value approach for grouping patients for survival analysis. PrognoScan provides a powerful platform for evaluating potential tumor markers and therapeutic targets and is publicly accessible at http://www.sabiosciences.com. The human IL-34 gene was used as an input source as a query and the data were collected for analysis.

\section{Results}

Comparative proteomic analysis of IL-34 protein identified in vertebrate genomes. All the IL-34 gene and protein sequences were collected from the Ensembl database and confirmed by BLAST at NCBI. The complete IL-34 gene was identified in the human, chimpanzee, gibbon, macaque, orangutan, marmoset, bushbaby, pika, squirrel, rat, mouse, kangaroo rat, elephant, cat, dog, panda, ferret, pig, horse, cow, flycatcher, chicken, zebrafish, platyfish and tilapia. The sequence and structural alignment of IL-34 is illustrated in Fig. 1. The phylogenetic tree was constructed according to the protein coding sequences of IL-34 using the ML method (Fig. 2). The IL-34 gene from the primate lineage, rodent lineage and teleost lineage forms a species-specific cluster. The exon-intron information collected 


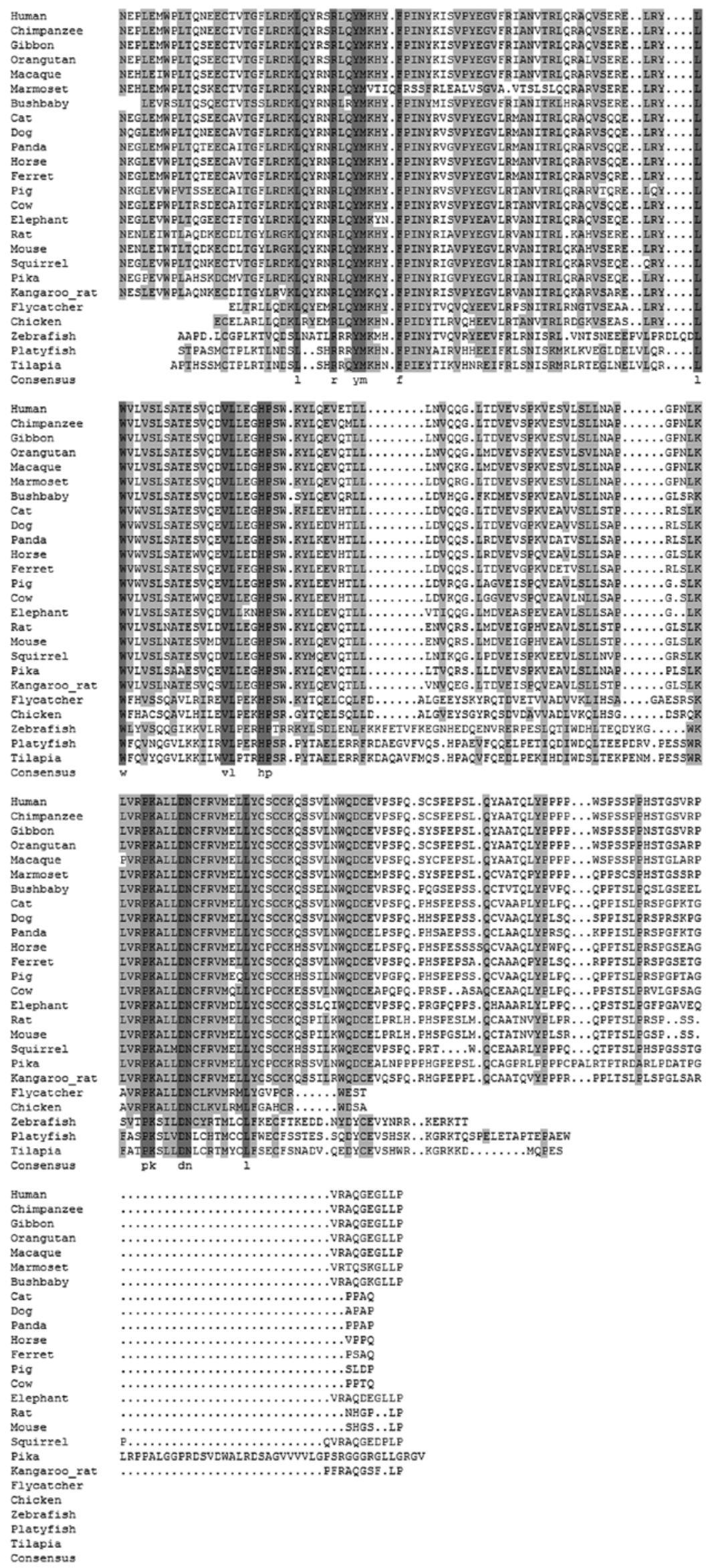

73
73
73
73
73
73
70
73
73
73
73
73
73
73
73
72
73
73
73
73
58
60
69
65
66

137

137

137

137

137

134

137

137

137

Figure 1. Sequence and structural alignment of vertebrate interleukin-34 (IL-34). All the IL-34 gene and protein sequences were collected from the Ensembl database and confirmed by BLAST at the National Center for Biotechnology Information (NCBI). The complete IL-34 gene was identified in 25 various mammalian genomes, such as the human, chimpanzee, gibbon, macaque, orangutan, marmoset, bushbaby, pika, squirrel, rat, mouse, kangaroo rat, elephant, cat, dog, panda, ferret, pig, horse, cow, flycatcher, chicken, zebrafish, platyfish and tilapia genomes. 


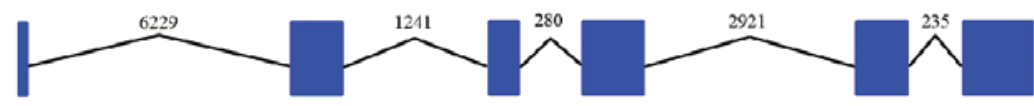

Mammalian

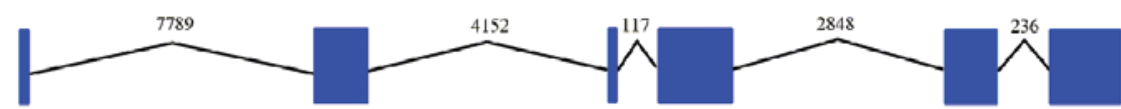

Marmoset

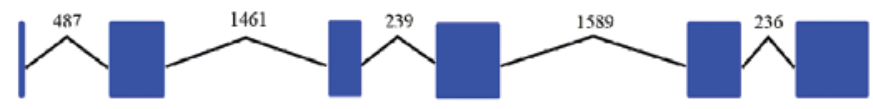

Bushbaby

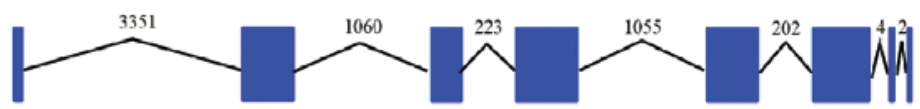

Kangaroo rat

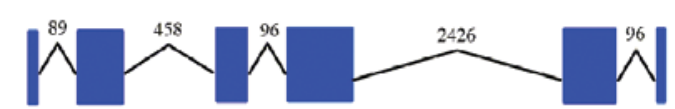

Bird

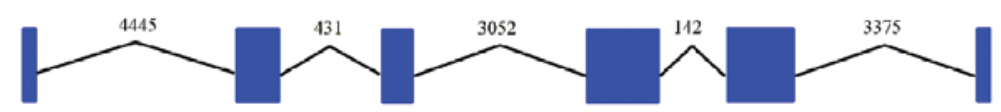

Zebrafish

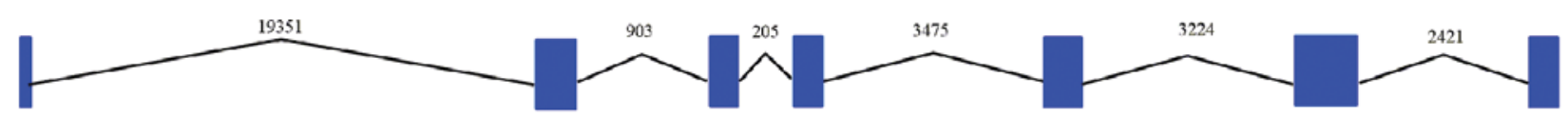

Platyfish and Tilapia

Figure 2. The phylogenetic tree was constructed according to the protein coding sequences of interleukin-34 (IL-34) using the maximum likelihood method. The IL-34 gene from the primate lineage, rodent lineage and teleost lineage formed a species-specific cluster.

from the Ensembl database is presented in Table I and Fig. 3. In the majority of genomes, the IL-34 gene has 6 exons with similar lengths in different species (Table I). In the majority of vertebrates, the IL-34 gene shows exon-intron conservation with 5 introns and similar sizes of each intron. With exception, there are 8 exons and 7 introns in the IL-34 gene in the kangaroo rat. Moreover, the IL-34 gene in the platyfish and tilapia contains 7 exons and 6 introns. Thus, the intron deletions of the IL-34 gene may occur during the evolutionary process in fish. Furthermore, site-specific tests for positive selection were performed for vertebrate, mammalian, primate and mammalian excluding primate, rodent and teleost lineages. Although some positive selection sites were computed, only the $2 \Delta \mathrm{l}$ of M7 and M8 of mammalian IL-34 was $>5.99$, indicating that the M8 model was more efficient than the M7 model in fitting the data. It seemed that mammalian IL-34 was under positive selecetion pressure with the identified positively selected site, 196Val (Table II).

Expression profile of the human IL-34 gene. By EST sequence searching, the human IL-34 gene was found to be expressed in the adult and fetal brain, the hippocampus, spleen, embryonic stem cells, heart, medulla, lung, testes, ovaries, metastatic chondrosarcoma, epidermis, keratinocytes, osteoarthritic cartilage, adipose tissue, choroid, eyes, amygdala, kidneys, thymus, small intestine, hypothalamus, islets of Langerhans, glioblastoma and the retinal pigment epithelium. The investigation of available microarray analyses and 'virtual northern blot analysis' revealed a predominant expression of IL-34 in the lymph nodes, brain, heart, skeletal muscle, colon, adipocyte, kidneys, liver, lungs, thyroid, adrenal gland, ovaries, prostate and testes. When performing a search in the PrognoScan database, the 


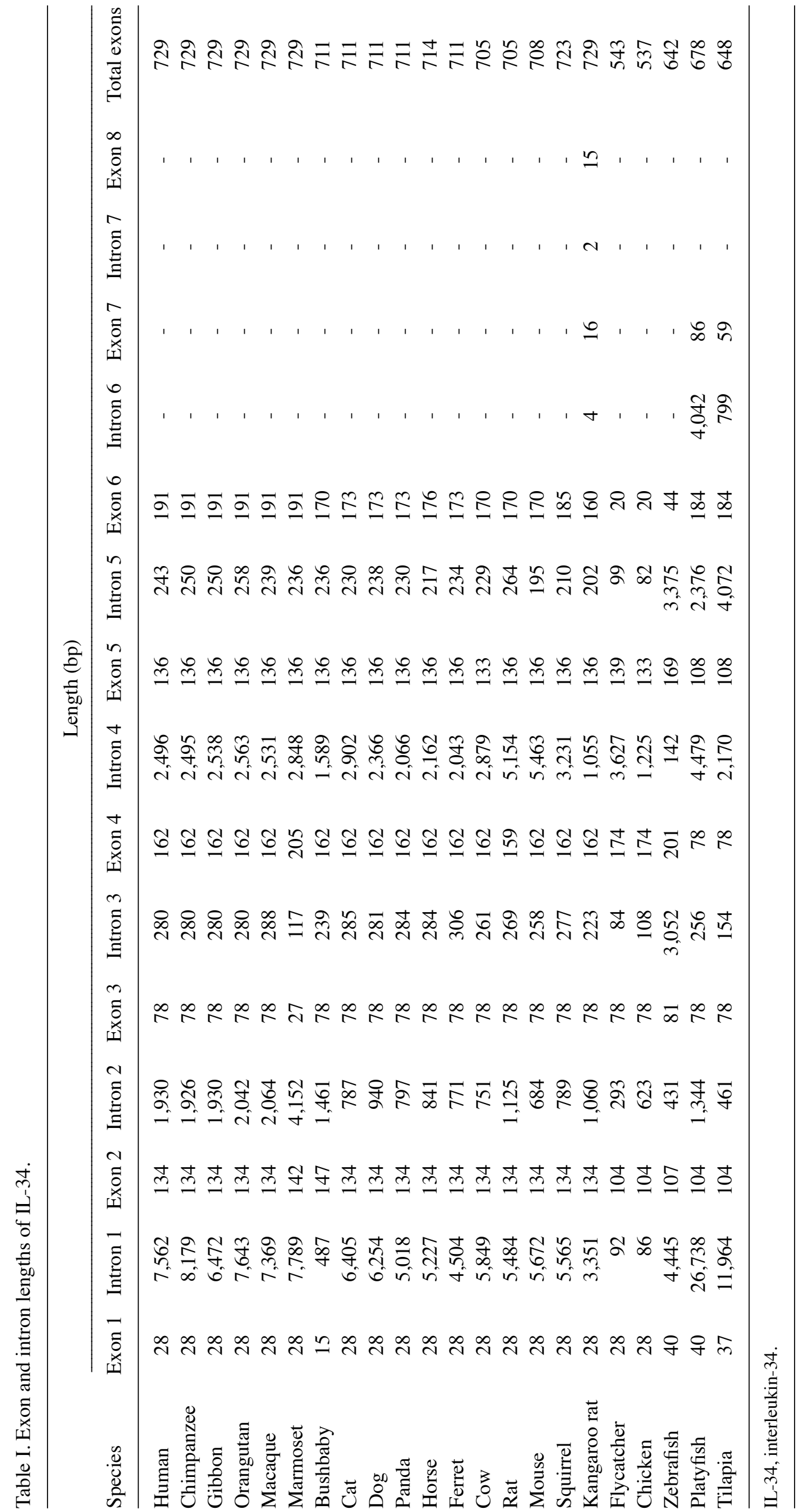


Table II. Site-specific tests for positive selection on IL-34.

\begin{tabular}{|c|c|c|c|c|c|}
\hline Species & Models & Estimates of parameters & $\ln L$ & $2 \Delta 1$ & $\begin{array}{c}\text { Positively } \\
\text { selected sites }\end{array}$ \\
\hline Vertebrate & $\begin{array}{l}\text { M7 } \\
\text { M8 }\end{array}$ & $\begin{array}{l}P=1.03903, Q=5.39585 \\
P 0=0.98039, P=1.20110, Q=7.10944 \\
(P 1=0.01961) w=1.00000\end{array}$ & $\begin{array}{l}-4659.912970 \\
-4657.855288\end{array}$ & 2.057682 & NS \\
\hline Mammalian & $\begin{array}{l}\text { M7 } \\
\text { M8 }\end{array}$ & $\begin{array}{l}P=0.48941, Q=1.45186 \\
P 0=0.96625, P=0.63368, Q=2.34086 \\
(P 1=0.03375), w=1.81330\end{array}$ & $\begin{array}{l}-4490.139825 \\
-4483.315416\end{array}$ & 6.824409 & $196 \mathrm{~V}^{\mathrm{a}}$ \\
\hline Primate & $\begin{array}{l}\text { M7 } \\
\text { M8 }\end{array}$ & $\begin{array}{l}P=0.01895, Q=0.02238 \\
P 0=0.56073, P=0.00997, Q=0.16501 \\
(P 1=0.43927) w=1.00000\end{array}$ & $\begin{array}{l}-1715.925824 \\
-1715.959612\end{array}$ & 0.033788 & NS \\
\hline $\begin{array}{l}\text { Mammalian excluding } \\
\text { primate }\end{array}$ & $\begin{array}{l}\text { M7 } \\
\text { M8 }\end{array}$ & $\begin{array}{l}P=0.30844, Q=1.06769 \\
P 0=0.98088, P=0.35332, Q=1.40393 \\
(P 1=0.01912) w=2.08098\end{array}$ & $\begin{array}{l}-3530.180011 \\
-3528.019777\end{array}$ & 2.160234 & $200 \mathrm{Q}^{\mathrm{a}}$ \\
\hline Rodent & $\begin{array}{l}\text { M7 } \\
\text { M8 }\end{array}$ & $\begin{array}{l}P=0.33256, Q=1.26857 \\
P 0=0.99999, P=0.33225, Q=1.26708 \\
(P 1=0.00001) w=3.17132\end{array}$ & $\begin{array}{l}-1743.972997 \\
-1743.973101\end{array}$ & 0.000104 & NS \\
\hline Teleost & $\begin{array}{l}\text { M7 } \\
\text { M8 }\end{array}$ & $\begin{array}{l}P=0.55893, Q=1.64564 \\
P 0=0.99243, P=0.58684, Q=1.81573 \\
(P 1=0.00757) w=8.77551\end{array}$ & $\begin{array}{l}-1656.009963 \\
-1655.921596\end{array}$ & 0.088367 & NS \\
\hline
\end{tabular}

${ }^{a}$ The positively selected sites were identified with posterior probability $\geq 0.95$ using the Bayes empirical Bayes (BEB) approach. $\operatorname{lnL}$, the $\log$-likelihood difference between the two models; $2 \Delta \mathrm{l}$, twice the log-likelihood difference between the two models (in all species, $2 \Delta \mathrm{l}<9.21$, the P-value is more than the significance level 0.05, indicating that the M8 model was more efficient than the M7 model); NA, not allowed; NS, not shown (sites under positive selection did not reach the significance level of 0.95). IL-34, interleukin-34.

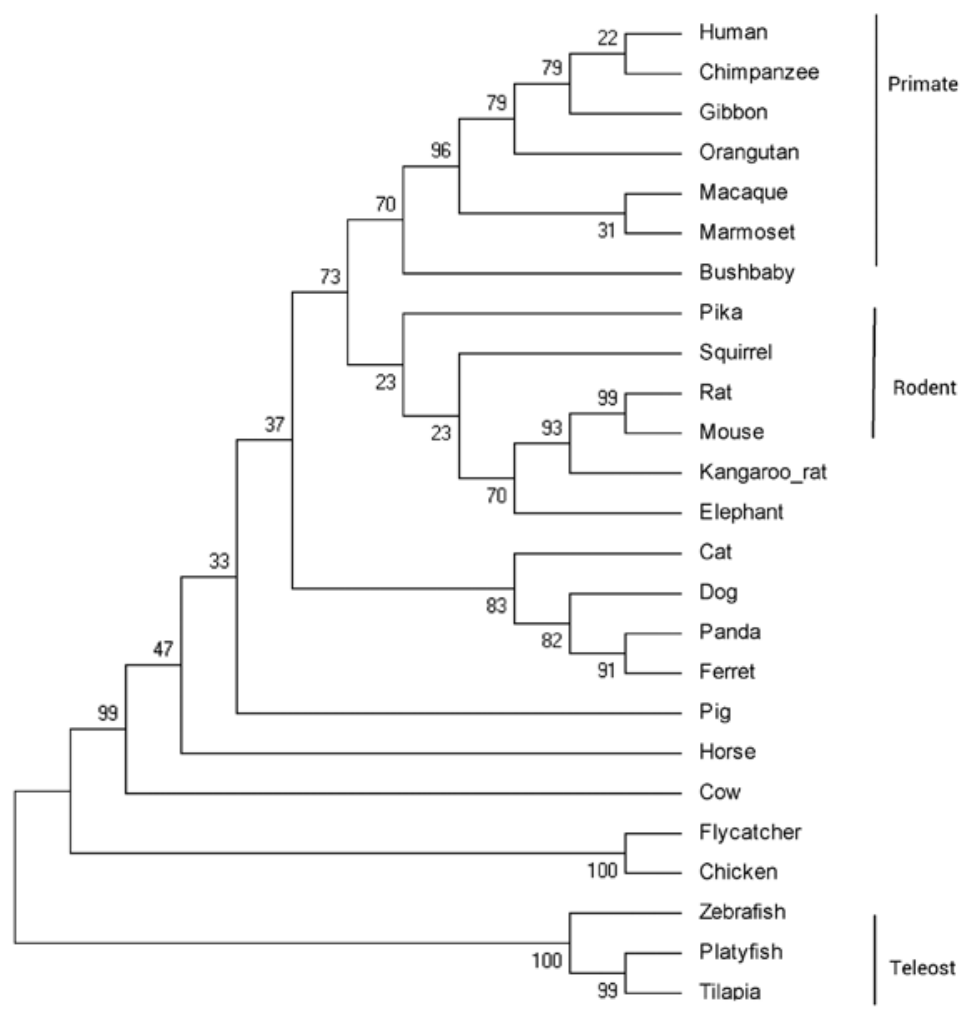

Figure 3. Exon-intron conservation of the interleukin-34 (IL-34) gene among different species. In the majority of vertebrates, the IL-34 gene showed an exonintron conservation with 5 introns and similar sizes of each intron. With exception, there are 8 exons and 7 introns in the IL-34 gene in the kangaroo rat. Moreover, in platyfish and tilapia, the IL-34 gene contains 7 exons and 6 introns. 
Table III. Functionally relevant SNP evaluation of the human IL-34 gene.

\begin{tabular}{|c|c|c|c|c|}
\hline SNP ID & $\begin{array}{l}\text { Chr } 16 \text { position } \\
\text { sequence }\end{array}$ & Sequence & Type & $\begin{array}{l}\text { Amino acid } \\
\text { change }\end{array}$ \\
\hline rs200158701 & 70680854(+) & CCATGC/TCCCGG & mis & PS \\
\hline rs 192337001 & $70680866(+)$ & GCTTCA/CCCTGG & mis & $\mathrm{TP}$ \\
\hline rs139133476 & $70688459(+)$ & CCTTGG/CCGTGG & mis & $\mathrm{AG}$ \\
\hline rs142890682 & $70688461(+)$ & TTGGCG/ATGGCC & mis & MV \\
\hline rs 118062333 & 70690511(+) & ААCACT/CACTTC & $\operatorname{mis}$ & HY \\
\hline rs200597979 & $70690960(+)$ & GGGCCA/GCCCAT & mis & HR \\
\hline rs8046424 & $70690989(+)$ & AGGTGC/GAGACG & mis & $\mathrm{QE}$ \\
\hline rs187166563 & $70693576(+)$ & CCCAGA/GGCCAA & mis & EG \\
\hline rs142214904 & $70693626(+)$ & GCTTCC/TGGGTC & mis & RW \\
\hline rs7206509 & $70693945(+)$ & GCCAAG/CTCCTC & mis & TS \\
\hline rs201277640 & 70693984(+) & GTATGC/TGGCCA & mis & AV \\
\hline rs202122982 & 70694001(+) & TGTACC/TCTCCG & mis & PS \\
\hline rs 148286339 & $70694011(+)$ & GCCCCC/TGTGGT & mis & PL \\
\hline rs141513638 & $70694056(+)$ & GAGGCC/TGGTCA & mis & PL \\
\hline rs112639369 & $70694073(+)$ & AGGGCG/AAGGGC & mis & $\mathrm{KE}$ \\
\hline rs1444643201 & 70694076(+) & GCGAGG/AGCCTC & mis & SG \\
\hline rs367851338 & $70693627(+)$ & CTTCCA/GGGTCA & mis & QR \\
\hline rs368143418 & $70690933(+)$ & TGAGTC/TGGTGC & $\operatorname{mis}$ & SL \\
\hline rs 374665339 & $70690963(+)$ & ССАСCC/ТАТССТ & $\operatorname{mis}$ & PL \\
\hline rs368923655 & $70691023(+)$ & CCTCAC/TGGTGA & $\operatorname{mis}$ & $\mathrm{TM}$ \\
\hline rs368367274 & $70693597(+)$ & GGTGCA/GGCCCA & mis & QR \\
\hline rs200891924 & $70693560(+)$ & TGTCCC/ATCTTG & mis & $\mathrm{IL}$ \\
\hline rs372998917 & $70694041(+)$ & CTCCAC/TGGGCT & mis & TM \\
\hline rs370436386 & $70690927(+)$ & TGCCAC/TTGAGT & mis & $\mathrm{TL}$ \\
\hline rs144427482 & $70690571(+)$ & CCAACG/ATCACC & mis & IV \\
\hline rs201108464 & $70693569(+)$ & TGAATG/ACCCCA & mis & TA \\
\hline rs144144426 & $70690541(+)$ & GTGTGC/TCTTAC & mis & PS \\
\hline rs377411431 & $70690885(+)$ & CGAGCG/TGGAGC & mis & RL \\
\hline rs369011177 & $70680875(+)$ & GGCTGC/TGCTGT & mis & $\mathrm{RC}$ \\
\hline rs 145782768 & $70693979(+)$ & TTGCAG/CTATGC & $\operatorname{mis}$ & HQ \\
\hline rs201784459 & $70694005(+)$ & СCCTCC/TGCCCC & mis & PL \\
\hline rs200488835 & $70693655(+)$ & TCCTGC/GTGTAA & mis & $\mathrm{CW}$ \\
\hline rs3813904 & 70680744(+) & TGACTG/CAGTGA & ese & \\
\hline rs3813905 & $70680850(+)$ & ACCACC/GATGCC & ese & \\
\hline rs4985556 & $70694000(+)$ & CTGTAC/ACCTCC & ese & \\
\hline
\end{tabular}

A total of 55 SNPs were functionally relevant; including 32 SNPs causing missense mutations, 3 exonic splicing enhancer SNPs and 20 SNPs causing nonsense mutations. SNP, single nucleotide polymorphism; IL-34, interleukin-34; mis, missense; ese, exonic splicing enhancer.

human IL-34 gene was also found to be expressed in various types of cancer, such as blood, brain, breast, colorectal, eye, head and neck, lung, ovarian and skin cancer.

Comparative genomic anlaysis of the human IL-34 gene. The upstream transcription factor 1 (USF1), regulatory factor X-1 (RFX1), the Sp1 transcription factor 1, POU class 3 homeobox 2 (POU3F2) and the forkhead box L1 (FOXL1) regulatory transcription factor binding sites were identified in the IL-34 gene upstream (promoter) region.
Functionally relevant SNP evaluation of the human IL-34 gene and identification of somatic mutations in human cancer. A total of 2,141 available SNPs were identified in the human IL-34 gene. Among these SNPs, a total of 55 SNPs were functionally relevant; these included 32 SNPs causing missense mutations, 3 exonic splicing enhancer SNPs and 20 SNPs causing nonsense mutations (Table III). As presented in Table IV, by performing a search of the COSMIC database, we identified 18 somatic mutations of the IL-34 gene in cancer. 
Table IV. Somatic mutations of IL-34 in cancer tissue.

\begin{tabular}{|c|c|c|c|c|c|}
\hline $\begin{array}{l}\text { Position } \\
\text { (AA) }\end{array}$ & $\begin{array}{l}\text { Mutation } \\
\text { (CDS) }\end{array}$ & $\begin{array}{c}\text { Mutation } \\
\text { (amino acid) }\end{array}$ & $\begin{array}{l}\text { Mutation ID } \\
\text { (COSM) }\end{array}$ & Count & Mutation type \\
\hline 4 & c. $11 \mathrm{G}>\mathrm{A}$ & p.G4D & COSM973055 & 1 & Substitution - missense \\
\hline 9 & c. $25 \mathrm{C}>\mathrm{T}$ & p.R9C & COSM3691133 & 1 & Substitution - missense \\
\hline 33 & c. $99 \mathrm{G}>\mathrm{A}$ & p.E33E & COSM704311 & 1 & Substitution - coding silent \\
\hline 38 & c. $114 \mathrm{G}>\mathrm{A}$ & p.T38T & COSM973057 & 1 & Substitution - coding silent \\
\hline 42 & c. $125 \_126 \mathrm{GG}>\mathrm{AA}$ & p.R42Q & COSM143555 & 1 & Substitution - missense \\
\hline 59 & c. $176 \mathrm{C}>\mathrm{T}$ & p.P59L & COSM108032 & 1 & Substitution - missense \\
\hline 61 & c. $182 \mathrm{~A}>\mathrm{G}$ & p.N61S & COSM3387573 & 1 & Substitution - missense \\
\hline 104 & c. $311 \mathrm{C}>\mathrm{T}$ & p.S104L & COSM435667 & 1 & Substitution - missense \\
\hline 155 & c. $465 \mathrm{C}>\mathrm{T}$ & p.N155N & COSM328594 & 9 & Substitution - coding silent \\
\hline 170 & c. $.508 \mathrm{C}>\mathrm{T}$ & p.R170W & COSM194870 & 1 & Substitution - missense \\
\hline 183 & c. $549 \mathrm{C}>\mathrm{A}$ & p.S183R & COSM3402448 & 1 & Substitution - missense \\
\hline 197 & c. $589 \mathrm{C}>\mathrm{T}$ & p.Q197* & COSM1379321 & 1 & Substitution - nonsense \\
\hline 197 & c. $590 A>G$ & p.Q197R & COSM1379322 & 1 & Substitution - missense \\
\hline 197 & c. $591 \mathrm{G}>\mathrm{A}$ & p.Q197Q & COSM40324 & 1 & Substitution - coding silent \\
\hline 208 & c. $623 \mathrm{C}>\mathrm{T}$ & p.A208V & COSM417292 & 2 & Substitution - missense \\
\hline 208 & c. $624 \mathrm{G}>\mathrm{A}$ & p.A208A & COSM1177412 & 1 & Substitution - coding silent \\
\hline 217 & c. $651 \mathrm{G}>\mathrm{A}$ & p.P217P & COSM1379323 & 1 & Substitution - coding silent \\
\hline 229 & c. $686 \mathrm{C}>\mathrm{T}$ & p.S229L & COSM417291 & 3 & Substitution - missense \\
\hline
\end{tabular}

IL-34, interleukin-34.

Meta-analysis of the prognostic value of the human IL-34 gene in cancer. When the name of a gene is submitted, PrognoScan displays a summary in table format of tests for the gene with columns for dataset, cancer type, subtype, endpoint, cohort, contributor, array type, probe ID, number of patients, optimal cutpoint, Pmin and Pcor. Among the databases which detected the expression of the IL-34 gene, 5 out of 40 tests revealed an association between the expression of the IL-34 gene and cancer prognosis (blood cancer, 1/4; brain cancer, 1/4; breast cancer, $0 / 11$; colorectal cancer, $1 / 7$; eye cancer, $0 / 1$; head and neck cancer, $0 / 3$; lung cancer, $2 / 6$; ovarian cancer, $0 / 3$; and skin cancer, $0 / 1$ ) with a $5 \%$ significance level (Table V). Among the two types of lung cancer, the lower expression of the IL-34 gene was related to poor survival and was found in non-small cell lung cancer (NSCLC) case (GSE8894). However, a higher expression of the IL-34 gene was related to poor survival in a case of adenocarcinoma (GSE31210). As for blood cancer cases and colorectal cancer, we found that a lower expression of the IL-34 gene was associated with poor survival. However, in the brain cancer cases, a higher expression of the IL-34 gene was related to poor survival.

\section{Discussion}

IL-34 was identified by functional screening of a library of secreted proteins, based on its ability to support human monocyte survival and to promote, with the same efficiency as M-CSF, the formation of the colony forming unit-macrophage (CFU-M) in human bone marrow cell cultures (4).
In the present study, we identified the complete IL-34 gene in 25 various mammalian genomes, including the human, chimpanzee, gibbon, macaque, orangutan, marmoset, bushbaby, pika, squirrel, rat, mouse, kangaroo rat, elephant, cat, dog, panda, ferret, pig, horse, cow, flycatcher, chicken, zebrafish, platyfish and tilapia genomes. In addition, we found that IL-34 existed in all types of vertebrates, including fish, amphibians, birds and mammals. The IL-34 gene has a similar 7 exon/6 intron gene organization in various species, and genes in the IL-34 loci were syntenically conserved $(33,34)$. The phylogenetic tree demonstrated that the IL-34 gene from the primate lineage, rodent lineage and teleost lineage formed a species-specific cluster. From the alignment and phylogenetic tree, mammalian IL-34 was conversed among vertebrate genomes, suggesting that the function of the IL-34 gene plays an important physiological role in all vertebrates in the long evolutionary process. It seemed that the mammalian IL-34 gene was under positive selection pressure with the identified positively selected site, 196Val. This is in accordance the with multiple biological functions of a cytokine, which plays a key role in the immune system.

IL-34 mRNA is widely expressed in various types of tissue, including tissue of the heart, brain, lung, liver, kidneys, thymus and spleen (4). Accordingly, by EST sequence searching, the IL-34 gene was also found to be expressed in various other types of tissues and cells, including the hippocampus, embryonic stem cells, medulla, testes, ovaries, metastatic chondrosarcoma, epidermis, keratinocytes, osteoarthritic cartilage, adipose tissue, choroid, eyes, amygdala thymus, 


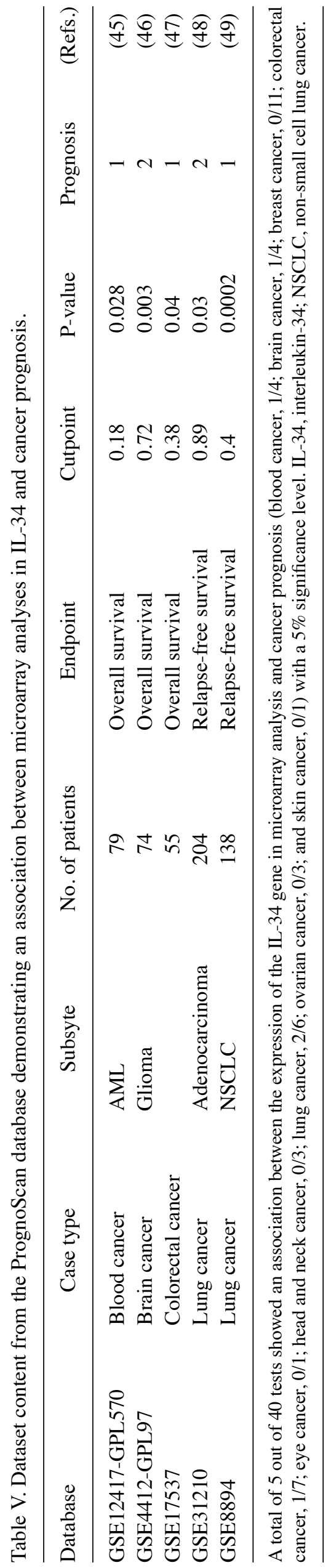

small intestine, hypothalamus, islets of Langerhans, glioblastoma and the retinal pigment epithelium. This suggests that the IL-34 gene is widely expressed in many types of tissues and organs. The investigation of available microarray analyses and 'virtual northern blot analysis' confirmed the predominant expression of IL-34 in the lymph nodes, brain, heart, skeletal muscle, colon, adipocyte, kidneys, liver, lung, thyroid, adrenal gland, ovaries, prostate and testes. A total of 55 functionally relevant SNPs, including 32 SNPs causing missense mutations, 3 exonic splicing enhancer SNPs and 20 SNPs causing nonsense mutations were identified from 2,141 available SNPs in the human IL-34 gene, which may affect the multiple functions of IL-34. However, the effects of these SNPs on the physiological and pathological function of IL-34 require further investigation.

IL-34 and M-CSF both signal via the same receptor, the M-CSF receptor, c-FMS. It has been shown that M-CSF is expressed at high levels in many types of tumor, including breast cancer, ovarian cancer and colorectal carcinoma and correlates with a poor prognosis (10-15). However, studies on the role of IL-34 in tumor development are limited. In the present study, we firstly found that IL-34 was indeed expressed in various types of cancer, such as blood, brain, breast, colorectal, eye, head and neck, lung, ovarian and skin cancer. A total of 5 out of 40 tests ( 1 blood cancer, 1 brain cancer, 1 colorectal cancer and 2 lung cancer) revealed an association between IL-34 gene expression and cancer prognosis. The mechanisms responsible for the involvement of IL-34 in the progression of these tumors require further investigation. It should be noted that the association between the expression of IL-34 and prognosis varies in different types of cancer, even in the same type of cancer from different databases. This suggests that the function of IL-34 in these tumors may be multidimensional, not only functioning as a tumor inhibitor or promoter. Moreover, we identified 18 somatic mutations of IL-34 in cancer tissue in the present study. The mechanisms through which these mutations affect tumor formation require further investigation. These data suggest that IL-34, similar to $\mathrm{M}-\mathrm{CSF}$, is involved in tumor formation.

USF1, RFX1, Sp1, POU3F2 and FOXL1 regulatory transcription factor binding sites were identified in the IL-34 gene upstream (promoter) region. USF-1 is an important transcription factor that participates in glucose metabolism and tumorigenesis. It has a negative effect on cell proliferation in some cell types and stabilizes the p53 protein and promotes a transient cell cycle arrest, in the presence of DNA damage $(34,35)$. RFX1 is unique transcription factor that contains a highly conserved 76-amino-acid DNA binding domain. RFX1 can directly regulate CD44 expression $(36,37)$. This mechanism may contribute to the effects of RFX1 on the proliferation, survival and invasion of glioblastoma cells. $\mathrm{Sp} 1$ is a member of the $\mathrm{Sp} / \mathrm{K}$ rüppel-like factor (KLF) family of transcription factors that play a critical role in embryonic and early postnatal development, differentiation, cell cycle regulation and in multiple diseases, including cancer (38-41). POU domain transcription factors are present in a number of cell lineages where they perform various functions, either as ubiquitous regulators of 'housekeeping' genes, or as developmental- and lineagespecific coordinators of cell fate decisions (42). POU3F2 has been shown to be responsive to MAPK pathway activation 
and to modulate the levels of microphthalmia-associated transcription factor (MITF) so as to suppress the differentiated melanocytic phenotype and to enhance tumor metastasis (29). FOXL1 is located at the junction of multiple signaling pathways and plays critical roles in a variety of physiological and pathological processes, including cancer development. These tumor-related transcriptional factors may be involved in the effects of IL-34 in tumors $(28,43,44)$.

\section{Acknowledgements}

This study was sponsored by grants from the Chinese National Natural Science Foundation (81202077 and 810001329), the National Major Scientific and Technological Special Project for 'Significant New Drugs Development' (2011ZX09302-003-02), the Jiangsu Province Major Scientific and Technological Special Project (BM2011017) and a Project Funded by the Priority Academic Program Development of Jiangsu Higher Education Institutions.

\section{References}

1. Wakabayashi S, Yamaguchi K, Kumakura S, et al: Effects of anesthesia with sevoflurane and propofol on the cytokine/ chemokine production at the airway epithelium during esophagectomy. Int J Mol Med 34: 137-144, 2014.

2. Signorelli SS, Fiore V and Malaponte G: Inflammation and peripheral arterial disease: the value of circulating biomarkers (Review). Int J Mol Med 33: 777-783, 2014.

3. Clavel G, Thiolat A and Boissier MC: Interleukin newcomers creating new numbers in rheumatology: IL-34 to IL-38. Joint Bone Spine 80: 449-453, 2013.

4. Lin H, Lee E, Hestir K, Leo C, et al: Discovery of a cytokine and its receptor by functional screening of the extracellular proteome. Science 320: 807-811, 2008.

5. Boström EA and Lundberg P: The newly discovered cytokine IL-34 is expressed in gingival fibroblasts, shows enhanced expression by pro-inflammatory cytokines, and stimulates osteoclast differentiation. PLoS One 8: e81665, 2013.

6. Moon SJ, Hong YS, Ju JH, Kwok SK, Park SH and Min JK: Increased levels of interleukin 34 in serum and synovial fluid are associated with rheumatoid factor and anticyclic citrullinated peptide antibody titers in patients with rheumatoid arthritis. J Rheumatol 40: 1842-1849, 2013.

7. Hwang SJ, Choi B, Kang SS, et al: Interleukin-34 produced by human fibroblast-like synovial cells in rheumatoid arthritis supports osteoclastogenesis. Arthritis Res Ther 14: R14, 2012.

8. Chen Z, Buki K, Vääräniemi J, Gu G and Väänänen HK: The critical role of IL-34 in osteoclastogenesis. PLoS One 6: e18689, 2011.

9. El-Gamal MI, Anbar HS, Yoo KH and Oh CH: FMS kinase inhibitors: Current status and future prospects. Med Res Rev 33: 599-636, 2013

10. Burns CJ and Wilks AF: c-FMS inhibitors: a patent review. Expert Opin Ther Pat 21: 147-165, 2011.

11. Baud'huin M, Renault R, Charrier C, et al: Interleukin-34 is expressed by giant cell tumours of bone and plays a key role in RANKL-induced osteoclastogenesis. J Pathol 221: 77-86, 2010.

12. Petráčková M, Staněk L, Mandys V, Dundr P and Vonka V: Properties of bcr-abl-transformed mouse 12B1 cells secreting interleukin-2 and granulocyte-macrophage colony stimulating factor (GM-CSF): II. Adverse effects of GM-CSF. Int J Oncol 40 1915-1922, 2012

13. Fournier P, Aigner M and Schirrmacher V: Targeting of IL-2 and GM-CSF immunocytokines to a tumor vaccine leads to increased anti-tumor activity. Int J Oncol 38: 1719-1729, 2011.

14. Kitoh Y, Saio M, Gotoh N, et al: Combined GM-CSF treatment and M-CSF inhibition of tumor-associated macrophages induces dendritic cell-like signaling in vitro. Int J Oncol 38: 1409-1419, 2011.

15. Tada F, Abe M, Hirooka M, et al: Phase I/II study of immunotherapy using tumor antigen-pulsed dendritic cells in patients with hepatocellular carcinoma. Int J Oncol 41: 1601-1609, 2012.
16. Yang L, Luo Y and Wei J: Integrative genomic analyses on Ikaros and its expression related to solid cancer prognosis. Oncol Rep 24: 571-577, 2010.

17. Yang L, Luo Y, Wei J and He S: Integrative genomic analyses on IL28RA, the common receptor of interferon- $\lambda 1,-\lambda 2$ and $-\lambda 3$. Int J Mol Med 25: 807-812, 2010.

18. Yang L, Wei $\mathbf{J}$ and He S: Integrative genomic analyses on interferon- $\lambda \mathrm{s}$ and their roles in cancer prediction. Int J Mol Med 25: 299-304, 2010.

19. Yu H, Yuan J, Xiao C and Qin Y: Integrative genomic analyses of recepteur d'origine nantais and its prognostic value in cancer. Int J Mol Med 31: 1248-1254, 2013.

20. Wang M, Wei X, Shi L, Chen B, Zhao G and Yang H: Integrative genomic analyses of the histamine $\mathrm{H} 1$ receptor and its role in cancer prediction. Int J Mol Med 33: 1019-1026, 2014.

21. Wang B, Chen K, Xu W, Chen D, Tang W and Xia TS: Integrative genomic analyses of secreted protein acidic and rich in cysteine and its role in cancer prediction. Mol Med Rep 10: 1461-1468, 2014.

22. Kumar S, Nei M, Dudley J and Tamura K: MEGA: a biologistcentric software for evolutionary analysis of DNA and protein sequences. Brief Bioinform 9: 299-306, 2008.

23. Yang Z: PAML: a program package for phylogenetic analysis by maximum likelihood. Comput Appl Biosci 13: 555-556, 1997.

24. Yang Z, Nielsen R, Goldman N and Pedersen AM: Codonsubstitution models for heterogeneous selection pressure at amino acid sites. Genetics 155: 431-449, 2000.

25. Forbes SA, Bindal N, Bamford S, et al: COSMIC: mining complete cancer genomes in the Catalogue of Somatic Mutations in Cancer. Nucleic Acids Res 39: D945-D950, 2011.

26. Katoh Y and Katoh M: Integrative genomic analyses on GLI1: positive regulation of GLI1 by Hedgehog-GLI, TGF $\beta$-Smads, and RTK-PI3K-AKT signals, and negative regulation of GLI1 by Notch-CSL-HES/HEY, and GPCR-Gs-PKA signals. Int J Oncol 35: 187-192, 2009.

27. Katoh $\mathrm{M}$ and Katoh M: Integrative genomic analyses of WNT11: transcriptional mechanisms based on canonical WNT signals and GATA transcription factors signaling. Int J Mol Med 24: 247-251, 2009.

28. Katoh M and Katoh M: Transcriptional mechanisms of WNT5A based on NF- $\mathrm{BB}$, Hedgehog, TGF $\beta$, and Notch signaling cascades. Int J Mol Med 23: 763-769, 2009.

29. Katoh M and Katoh M: Integrative genomic analyses of ZEB2: Transcriptional regulation of ZEB2 based on SMADs, ETS1, HIF1 $\alpha$, POU/OCT, and NF-кB. Int J Oncol 34: 1737-1742, 2009.

30. Chalifa-Caspi V, Yanai I, Ophir R, et al: GeneAnnot: comprehensive two-way linking between oligonucleotide array probesets and GeneCards genes. Bioinformatics 20: 1457-1458, 2004.

31. Parkinson H, Sarkans U, Shojatalab M, et al: ArrayExpress - a public repository for microarray gene expression data at the EBI. Nucleic Acids Res 33: D553-D555, 2005.

32. Mizuno H, Kitada K, Nakai K and Sarai A: PrognoScan: a new database for meta-analysis of the prognostic value of genes. BMC Med Genomics 2: 18, 2009.

33. Wang T, Kono T, Monte MM, et al: Identification of IL-34 in teleost fish: differential expression of rainbow trout IL-34, MCSF1 and MCSF2, ligands of the MCSF receptor. Mol Immunol 53: 398-409, 2013.

34. Bouafia A, Corre S, Gilot D, Mouchet N, Prince S and Galibert MD: p53 requires the stress sensor USF1 to direct appropriate cell fate decision. PLoS Genet 10: e1004309, 2014.

35. Ikeda R, Nishizawa Y, Tajitsu Y, et al: Regulation of major vault protein expression by upstream stimulating factor 1 in SW620 human colon cancer cells. Oncol Rep 31: 197-201, 2014.

36. Katoh Y and Katoh M: Comparative genomics on Vangl1 and Vangl2 genes. Int J Oncol 26: 1435-1440, 2005.

37. Feng C, Zhang Y, Yin J, Li J, Abounader R and Zuo Z: Regulatory factor $\mathrm{X} 1$ is a new tumor suppressive transcription factor that acts via direct downregulation of CD44 in glioblastoma. Neuro Oncol 16: 1078-1085, 2014.

38. Xu F, Zhu X, Han T, et al: The oncoprotein hepatitis B $\mathrm{X}$-interacting protein promotes the migration of ovarian cancer cells through the upregulation of S-phase kinase-associated protein 2 by Sp1. Int J Oncol 45: 255-263, 2014.

39. Liang X, Li ZL, Jiang LL, Guo QQ, Liu MJ and Nan KJ: Suppression of lung cancer cell invasion by LKB1 is due to the downregulation of tissue factor and vascular endothelial growth factor, partly dependent on SP1. Int J Oncol 44: 1989-1997, 2014

40. Chae JI, Cho JH, Lee KA, et al: Role of transcription factor Sp1 in the quercetin-mediated inhibitory effect on human malignant pleural mesothelioma. Int J Mol Med 30: 835-841, 2012. 
41. Lee KA, Lee YJ, Ban JO, et al: The flavonoid resveratrol suppresses growth of human malignant pleural mesothelioma cells through direct inhibition of specificity protein 1 . Int $\mathrm{J}$ Mol Med 30: 21-27, 2012.

42. Moritsugu R, Tamai K, Nakano H, et al: Functional analysis of the nuclear localization signal of the POU transcription factor Skn-1a in epidermal keratinocytes. Int J Mol Med 34: 539-544, 2014.

43. Katoh M and Katoh M: Human FOX gene family (Review). Int J Oncol 25: 1495-1500, 2004.

44. Katoh $\mathrm{M}$ and Katoh M: Transcriptional regulation of WNT2B based on the balance of Hedgehog, Notch, BMP and WNT signals. Int J Oncol 34: 1411-1415, 2009.

45. Metzeler KH, Hummel M, Bloomfield CD, et al: An 86-probe-set gene-expression signature predicts survival in cytogenetically normal acute myeloid leukemia. Blood 112: 4193-4201, 2008.
46. Freije WA, Castro-Vargas FE, Fang Z, et al: Gene expression profiling of gliomas strongly predicts survival. Cancer Res 64: 6503-6510, 2004

47. Smith JJ, Deane NG, Wu F, et al: Experimentally derived metastasis gene expression profile predicts recurrence and death in patients with colon cancer. Gastroenterology 138: 958-968, 2010.

48. Okayama H, Kohno T, Ishii Y, et al: Identification of genes upregulated in ALK-positive and EGFR/KRAS/ALK-negative lung adenocarcinomas. Cancer Res 72: 100-111, 2012.

49. Lee ES, Son DS, Kim SH, et al: Prediction of recurrence-free survival in postoperative non-small cell lung cancer patients by using an integrated model of clinical information and gene expression. Clin Cancer Res 14: 7397-7404, 2008. 